\title{
Reduced Density Matrices of the Anisotropic Heisenberg Model *
}

\author{
J. GINIBRE \\ Department of Physics, New York University
}

Received May 2, 1968

\begin{abstract}
The reduced density matrices of the anisotropic Heisenberg model are studied by means of a functional integral representation based on a generalized Poisson process. Integral equations, which are analogous to the classical KirkwoodSalzburg equations, are then used to prove the existence of the infinite volume limit of the reduced density matrices, analyticity properties with respect to the fugacity (or magnetic field) and the potentials, and a cluster property, in the low fugacity (high magnetic field) region.
\end{abstract}

\section{Introduction}

The correlation functions of classical gases and their quantum analogues have been studied recently by a method due to RuELLE [1]. This method rests on the well known Kirkwood-Salzburg equations [2] and various generalizations thereof, and has been used to prove the existence of the infinite volume limit, some analyticity properties, and a cluster property of the correlation functions for sufficiently small fugacities. The method has been applied to classical continuous systems [1], to classical lattice systems [3], and to continuous quantum systems [4]. In the latter case, in order to obtain and exploit appropriate generalizations of the Kirkwood-Salzburg equations, one uses an integral representation of the quantum analogues of the correlation functions, namely the reduced density matrices (R.D.M.), based on the Wiener integral.

In the present paper, we want to point out that the same method also applies to a variety of quantum lattice systems. (For general properties of such systems, see ref. [5, 6, 7].) In fact, one can use a functional integral representation of the RDM based on a discrete analogue of the Wiener process, which turns out to be a generalized Poisson process. Various models can be studied, some of which are listed in the last section of this paper. Meanwhile, we concentrate on one of them, namely the anisotropic Heisenberg model, which is both of current interest and typical of the scope of the method.

In Section 1, we define the model, describe the associated stochastic process, and give some estimates which are needed in subsequent proofs. In Section 2, we give the definition and integral representation of the

$\star$ The research reported in this paper was supported by the National Science Foundation. 
RDM, and the appropriate generalizations of the Kirkwood-Salzburg equations, as well as upper bounds and analyticity properties of their kernels. We then state the results that can be obtained from them in Section 3. Most of the proofs are identical with those of the continuous quantum case [4] or of the classical lattice case [3] and are omitted. Finally, we compare the results for quantum lattices with those obtained in the other cases, and discuss briefly some related models that can be treated by the same method.

\section{The Model and the Associated Stochastic Process}

The anisotropic Heisenberg model can be thought of either as a spin system or as a lattice gas [8]. We shall use the latter formulation, which is more convenient for our purpose. The configuration space is a $v$-dimensional cubic lattice. At each lattice site $r$, there are defined boson field operators $a_{r}, a_{r}^{+}$with the canonical commutation relation $\left[a_{r}, a_{s}^{+}\right]=\delta_{r s}$, all other commutators being zero. We first consider a system enclosed in a finite box $\Lambda$ of volume (= number of sites) $\mathrm{V}$, which eventually will become infinite. The system is described in the grand canonical formalism. The hamiltonian is chosen as:

$$
\begin{aligned}
H= & -\frac{1}{4} \sum_{r, 8}\left(a_{r}^{+}-a_{s}^{+}\right)\left(a_{r}-a_{s}\right) \varphi_{\perp}(r-s)-\mu \sum_{r} a_{r}^{+} a_{r} \\
& +\frac{1}{2} \sum_{r, s} a_{r}^{+} a_{s}^{+} a_{r} a_{s} \varphi_{\|}(r-s) .
\end{aligned}
$$

The transverse potential $\varphi_{\perp}$ is supposed to be symmetric $\left(\varphi_{\perp}(r)=\varphi_{\perp}(-r)\right)$ and to satisfy:

We define also $M_{0}$ by

$$
\sum_{r \neq 0}\left|\varphi_{\perp}(r)\right|=2 M<\infty .
$$

$$
\sum_{r \neq 0} \varphi_{\perp}(r)=2 M_{0} .
$$

The longitudinal potential is supposed to have a hard core $\left(\varphi_{\|}(0)=+\infty\right)$, to be symmetric $\left(\varphi_{\|}(r)=\varphi_{\|}(-r)\right)$ and to satisfy:

$$
\sum_{r \neq 0}\left|\varphi_{\|}(r)\right|=\phi<\infty \text {. }
$$

If $\varphi_{\|}$is real, we define $\phi^{+}$and $\phi_{-}$by

$$
\phi_{ \pm}=\sum_{r \neq 0} \operatorname{Max}\left( \pm \varphi_{\|}(r), 0\right) .
$$

We shall be also interested in complex potentials. They form a Banach space $\mathscr{B}$ with the norm:

10 Commun. math. Phys., Vol. 10

$$
\|\varphi\|=\sum_{r \neq 0}|\varphi(r)| .
$$


The hard core condition reduces the Fock space of the system to the subspace of functions that vanish whenever two arguments coincide. One can therefore replace the $a, a^{+}$on each site by PAULI matrices $a^{+} \rightarrow \sigma^{+}=\left(\begin{array}{ll}0 & 1 \\ 0 & 0\end{array}\right), a \rightarrow \sigma^{-}=\left(\begin{array}{ll}0 & 0 \\ 1 & 0\end{array}\right)$ with modified commutations relations which take the hard core condition into account automatically. This gives immediately the hamiltonian in the usual spin system formulation:

$$
\begin{aligned}
H= & \frac{1}{8} \sum_{r \neq s}\left\{\left(\sigma_{r}^{x} \sigma_{s}^{x}+\sigma_{r}^{y} \sigma_{s}^{y}\right) \varphi_{\perp}(r-s)+\sigma_{r}^{z} \sigma_{s}^{z} \varphi_{\|}(r-s)\right\} \\
& -\frac{1}{2} h \sum_{r} \sigma_{r}^{z}-C
\end{aligned}
$$

where the magnetic field is related to the chemical potential by:

$$
h=\mu+\frac{1}{2} \sum_{r \neq 0}\left(\varphi_{\perp}(r)-\varphi_{\| \mid}(r)\right) .
$$

$C$ is a constant proportional to the volume:

$$
C=-\frac{1}{2} V\left(\mu+\frac{1}{2} \sum_{r}\left(\varphi_{\perp}(r)-\frac{3}{2} \varphi_{\|}(r)\right)\right)
$$

and we have omitted surface terms which are easily dealt with. We shall not make use of the magnetic formulation in the sequel.

We now concentrate on the first sum in (1.1), which we call $T$, and which is meant to represent the kinetic energy. This interpretation is borne out by the following argument. Let $\psi\left(r_{1}, \ldots, r_{n}\right)$ be a $n$-particle wave function. One then sees easily that:

$$
\begin{aligned}
& (T \psi)\left(r_{1}, \ldots, r_{n}\right)=\frac{1}{4} \sum_{s} \sum_{j=1}^{n}\left\{\psi\left(r_{1}, \ldots, r_{j}+s, \ldots, r_{n}\right)\right. \\
& \left.\quad+\psi\left(r_{1}, \ldots, r_{j}-s, \ldots, r_{n}\right)-2 \psi\left(r_{1}, \ldots, r_{n}\right)\right\} \quad \varphi_{\perp}(s) .
\end{aligned}
$$

The double differences are the natural analogues of the Laplace operator. It is essential for this interpretation of $T$ and the following considerations that the hard core condition be absent from $T$, or in other words, that the $a, a^{+}$be true boson operators and not Pauli matrices.

In the continuous case, the statistical operator $W=\exp (-\beta H)$ can be represented by means of the Wiener integral [9], which is associated in a natural way with the heat equation $\partial \psi / \partial t=\frac{1}{2} \Delta \psi$. We shall obtain a similar representation here, starting from the corresponding one particle equation:

$$
\frac{\partial \psi(r)}{\partial t}=-\frac{1}{2} \sum_{\boldsymbol{s}} \varphi_{\perp}(s)(\psi(r+s)-\psi(r))
$$

or:

$$
\frac{\partial \hat{\psi}(\theta)}{\partial t}=-\frac{1}{2} \sum_{\boldsymbol{s}} \varphi_{\perp}(s)\left(e^{i s \cdot \theta}-1\right) \hat{\psi}(\theta)
$$


where $\hat{\psi}(\theta)$ is the Fourier transform of $\psi$ :

$$
\hat{\psi}(\theta)=\sum_{r} \psi(r) e^{-i r_{\bullet} \theta} .
$$

The solution of (1.11) that reduces to $\delta_{0, r}$ for $t=0$ is then:

where:

$$
\psi_{t}(r)=(2 \pi)^{-v} \int_{0}^{2 \pi} d^{v} \theta \hat{\psi}_{t}(\theta) e^{i r . \theta}
$$

$$
\hat{\psi}_{t}(\theta)=\exp \left\{\frac{1}{2} t \sum_{\delta} \varphi_{\perp}(s)\left(1-e^{i s \cdot \theta}\right)\right\}
$$

or:

where:

$$
\hat{\psi}_{t}(\theta)=\exp \left\{t \int\left(e^{i x \cdot \theta}-1\right) d M(x)\right\}
$$

$$
d M(x)=-\frac{1}{2} \sum_{s \neq 0} \varphi_{\perp}(s) \delta(x-s)
$$

$d M$ is a discrete measure in the underlying $v$-dimensional euclidean space, with total variation $M$. We then define a stochastic process $\omega(t)$ in $R^{\nu}$ with stationary independent increments as follows.

We first consider the case where $\varphi_{\perp}(r) \leqq 0$ for all $r$. If $T$ is interpreted as a kinetic energy, this condition means that the "mass of the particle" is positive. The measure $d M$ is then positive. We define the process $\omega(t)$ by taking the distribution of the increment $\omega\left(t^{\prime}\right)-\omega(t)$ to be:

$$
d \mu(x)=\sum_{r} \psi_{\left|t^{\prime}-t\right|}(r) \delta(x-r) .
$$

(1.16) is then the Levy-Khinchine representation of the associated characteristic function [10], and shows that the process is a generalized Poisson process [10]. It can be described as follows: $\omega(t)$ is constant except for jumps. The number $n$ of jumps in any finite time interval of length $t$ is finite almost everywhere, with a Poisson distribution:

$$
P_{n}=e^{-M t} \frac{(M t)^{n}}{n !}
$$

$M$ is the average number of jumps per unit time. The probability distribution of the jumps is given by the measure $d M$. The possible values of the jumps are the vectors in the support of $d M$. They have therefore integral valued components (so that $\omega(t)$ remains on the lattice!), the conditional probability of jumping by $r$ being $-(2 M)^{-1} \varphi_{\perp}(r)$. The process will be normalized to have a fixed starting point: $\omega(0)=r$ with probability one. We shall always use finite time intervals. We call $P_{r 8}^{t}(d \omega)$ the measure on the set of paths starting from $r$ at time zero and ending at $s$ at time $t$. It is normalized by:

$$
\sum_{s} \int P_{r s}^{t}(d \omega)=1
$$


We now turn to the general case where $\varphi_{\perp}$ is not necessarily negative, and possibly complex, but still satisfies (1.2). To such a potential we associate a (non positive) measure $P_{r s}^{t}(d \omega)$ as follows: Let $P_{+r s}^{t}(d \omega)$ be the positive measure associated with the potential $-\left|\varphi_{\perp}(r)\right|$, as described above. We then define the measure $P_{r s}^{t}(d \omega)$ on the same sets of paths by the conditions:

(1) $P_{r s}^{t}(d \omega)$ is absolutely continuous with respect to $P_{+r s}^{t}(d \omega)$.

(2) Its Radon-Nikodym derivative $f_{t}(\omega)$ is defined almost everywhere by:

$$
f_{t}(\omega)=\exp \left[t\left(M+M_{0}\right)\right] \prod_{i=1}^{n} \frac{-\varphi_{\perp}\left(r_{i}\right)}{\left|\varphi_{\perp}\left(r_{i}\right)\right|}
$$

if the path $\omega$ has $n$ jumps of magnitude $r_{1}, \ldots, r_{n}$ in the time interval $(0, t)$. Here $M_{0}$ is defined by $(1.3)$.

The function $f_{t}(\omega)$ is identically equal to one if $\varphi_{\perp}(r)$ is real negative. It is bounded for all $\omega$. More precisely:

$$
\left|f_{t}(\omega)\right|=\exp \left[t\left(M+\operatorname{Re} M_{0}\right)\right] .
$$

We shall often make use of the relation:

$$
\left|P_{r s}^{t}(d \omega)\right|=\exp \left[t\left(M+\operatorname{Re} M_{0}\right)\right] P_{+r s}^{t}(d \omega) .
$$

The measure $P_{r s}^{t}(d \omega)$ thus defined bears the same relation to the Eq. (1.11) and its solution (1.14) as before; more precisely:

In fact, consider:

$$
\int P_{0 r}^{t}(d \omega)=\psi_{t}(r) \text {. }
$$

$$
\begin{gathered}
\sum_{r} \int P_{0 r}^{t}(d \omega) e^{i r \cdot \theta}=\sum_{r} \int P_{+0 r}^{t}(d \omega) f_{t}(\omega) e^{i r_{0} \theta} \\
=\sum_{n} e^{-M t} \frac{(M t)^{n}}{n !} \sum_{r} \sum_{\substack{r_{1}, \ldots ., r_{n} \\
\Sigma \Sigma_{i}=r}} \prod_{i=1}^{n} \frac{\left|\varphi_{\perp}\left(r_{i}\right)\right|}{2 M} \\
\quad \cdot \exp \left[t\left(M+M_{0}\right)\right] \prod_{i=1}^{n} \frac{-\varphi_{\perp}\left(r_{i}\right)}{\left|\varphi_{\perp}\left(r_{i}\right)\right|} e^{i r \cdot \theta} \\
\quad=\exp \left\{\frac{1}{2} t \sum_{r} \varphi_{\perp}(r)\left(1-e^{i} \cdot r_{0} \theta\right)\right\}=\hat{\psi}_{t}(\theta) .
\end{gathered}
$$

We shall need later on an upper bound for the function:

$$
I(\tau)=\sum_{r} \int\left|P_{0 r}^{t}(d \omega)\right| \exp (\tau n(\omega))
$$

where $\tau$ is any real number, and $n(\omega)$ is the number of jumps of $\omega$ in the time interval $(0, t)$. It is easily seen that:

$$
\begin{aligned}
I(\tau) & =\exp \left(t \operatorname{Re} M_{0}\right) \sum_{n} \frac{(M t)^{n}}{n !} e^{\tau n} \\
& =\exp \left[t\left(M e^{\tau}+\operatorname{Re} M_{0}\right)\right] .
\end{aligned}
$$


We shall also make use of the following upper bound:

$$
\begin{aligned}
& \operatorname{Sup}_{r} \int\left|P_{0 r}^{t}(d \omega)\right| \exp [\tau n(\omega)] \\
& \leqq e^{t \operatorname{Re} M_{0}} \sum_{n} \frac{(M t)^{n}}{n !} e^{\tau n} \operatorname{Sup}_{r} \sum_{\substack{r_{1}, \ldots, r_{n} \\
\Sigma r_{i}=r}} \prod_{i} \frac{\left|\varphi_{\perp}\left(r_{i}\right)\right|}{2 M} \\
& \leqq \alpha \exp \left[t\left(M e^{\tau}+\operatorname{Re} M_{0}\right)\right]+(1-\alpha) \exp \left(t \operatorname{Re} M_{0}\right), \\
& \leqq \exp \left[t\left(M e^{\tau}+\operatorname{Re} M_{0}\right)\right]
\end{aligned}
$$

where:

$$
\alpha=(2 M)^{-1} \operatorname{Sup}_{r}\left|\varphi_{\perp}(r)\right|(\leqq 1 / 2) .
$$

We are now able to represent the statistical operator $W=\exp (-\beta H)$ as an integral in the space of the paths of the previous process. The result is a transcription in the lattice case of the Wiener integral representation $[9,4]$ which holds in the continuous case. The proof will be omitted. $H$ commutes with the particle number operator. In the $m$ particle space, $W$ is represented by an integral kernel $W\left(r^{m}, s^{m}\right)$ where $r^{m}=r_{\mathbf{1}}, \ldots, r_{m}$ and $s^{m}=s_{1}, \ldots, s_{m}$ are two families of $m$ points of the lattice. Then:

$$
W\left(r^{m}, s^{m}\right)=\int_{\Lambda} P_{r^{m}, s^{m}}^{\beta}\left(d \omega^{m}\right) z^{m} \exp \left[-U\left(\omega^{m}\right)\right] .
$$

Here $\omega^{m}$ is a set of $m$ paths: $\omega^{m}=\omega_{1}, \ldots, \omega_{m}$, going from $r^{m}$ to $s^{m}$. $z=\exp (\beta \mu)$ is the fugacity, and the interaction is given by:

$$
U\left(\omega^{m}\right)=\int_{0}^{\beta} d t\left\{\sum_{i<j} \varphi_{\|}\left(\omega_{i}(t)-\omega_{j}(t)\right)\right\} .
$$

The fact that the system is enclosed in the box $\Lambda$ appears through the restriction that all paths are contained in $\Lambda$.

\section{The RDM and the KS Equations}

In this section, we give the definition and integral representation of the RDM, write down the KS equations and derive upper bounds and analyticity properties for their kernels, as an illustration of the method of proof of the subsequent results.

We consider first a finite system. Let $Z_{A}=\operatorname{Tr} W$ and $\langle A\rangle$ $=Z_{\Lambda}^{-1} \operatorname{Tr}(A W)$. The RDM are then defined by:

where:

$$
\bar{\varrho}_{\Lambda}\left(r^{m}, s^{m}\right)=\left\langle a^{+}\left(s^{m}\right) a\left(r^{m}\right)\right\rangle
$$

$$
a\left(r^{m}\right)=\prod_{i=1}^{m} a_{r_{i}}
$$

and similarly for $a^{+}$. The hard core implies that $\bar{\varrho}_{A}=0$ whenever for some $(i, j), r_{i}=r_{j}$ or $s_{i}=s_{j}$. In the spin language, one would replace $a^{+}$ 
and $a$ by $\sigma^{+}$and $\sigma^{-}$, and express the products $\sigma^{+} \sigma^{-}$for those points common to $r^{m}$ and $s^{m}$ in terms of $\sigma^{z}$. The RDM can be represented as follows. We call length of a path the length of its time interval. We shall need elementary paths of length $\beta$, and composite paths of length an integer multiple of $\beta$, obtained by piecing several elementary paths together. Then:

$$
\bar{\varrho}_{\Lambda}\left(r^{m}, s^{m}\right)=\mathscr{S} \prod_{i=1}^{m}\left\{\sum_{j_{i} \geqq 1} \int P_{r_{i}, s_{i}}^{j_{i} \beta}\left(d \omega_{i}\right)\right\} \varrho_{\Lambda}(X)
$$

where $\mathscr{S}$ means sum over all permutations of the variables $r$ (or $s$ ). $X=\omega_{1}, \ldots, \omega_{m}$ is a family of $m$ composite paths of respective lengths $j_{i} \beta \cdot \varrho_{\Lambda}(X)$ is a function of these $m$ paths, defined as follows:

$$
\varrho_{\Lambda}(X)=Z_{\Lambda}^{-1} f d Y z^{p+q} \exp [-U(X+Y)] \alpha_{\Lambda}(X+Y)
$$

with the following notations: $Y=\bar{\omega}_{1}, \ldots, \bar{\omega}_{n}$ is a family of $n$ composite closed paths. $p$ and $q$ are the total number of elementary paths that build the composite paths in $X$ and $Y$ respectively. The integration symbol is defined as:

where:

$$
f d Y=\sum_{n=0}^{\infty}(n !)^{-1} \int d \bar{\omega}_{1} \ldots d \bar{\omega}_{n}
$$

$$
\int d \bar{\omega}=\sum_{j=1}^{\infty} \sum_{r} \frac{1}{j} \int P_{r r}^{j \beta}(d \bar{\omega}) .
$$

$U(X+Y)$ is defined as in (1.33), the sum now running over all the elementary paths that build $X$ and $Y$. Finally, $\alpha_{A}$ is 1 if all the paths are contained in $\Lambda$ and zero otherwise. $X+Y$ represents the union of $X$ and $Y$.

The proof is the same as in the continuous case [4]. The $\varrho_{\Lambda}(X)$ are easily seen to satisfy the following generalizations of the K.S. equations:

$$
\begin{gathered}
\varrho_{\Lambda}(X+\omega)=\alpha_{\Lambda}(X+\omega) z \exp [-F(\omega, X)] f d Y K(\omega, Y) \\
\cdot \varrho_{\Lambda}(X+Y)
\end{gathered}
$$

where $\omega$ is an elementary path, and where:

$$
F(\omega, X)=\int_{0}^{\beta} d t \sum_{i=1}^{p} \varphi_{\|}\left(\omega(t)-\omega_{i}(t)\right) .
$$

The sum runs over all elementary paths that build $X$.

where:

$$
K(\omega, Y)=\prod_{i=1}^{n} K\left(\omega, \bar{\omega}_{i}\right)
$$

$K(\omega, \bar{\omega})=\exp \left[-\int_{0}^{\beta} d t \sum_{k=1}^{j} \varphi_{\|}(\omega(t)-\bar{\omega}(t+(k-1) \beta))\right]-1$

if $\bar{\omega}$ is a composite path of length $j \beta$. 
The proof is the same as in the continuous case [4]. The K.S. equations can be considered as a linear equation in the space $\mathscr{E}$ of sequences of complex functions of families of an increasing number of elementary or composite paths (a composite path can always be regarded as a family of elementary paths) that vanish whenever two of these elementary paths have overlapping hard cores, or more precisely spend a common finite time interval on the same lattice site. The family of the $\varrho_{A}(X)$ define a vector $\varrho_{A}$ in $\mathscr{E}$ and the Eq. (2.7) is then of the form:

$$
\varrho_{\Lambda}=A_{\Lambda}\left(\zeta+z \mathscr{K} \varrho_{\Lambda}\right)
$$

where $A_{\Lambda}$ is multiplication by $\alpha_{\Lambda}$, i.e. restriction of the functions to the set of paths that stay in $A$. $\zeta$ is the vector in $\mathscr{E}$ defined by $\zeta(\omega)=z$ if $\omega$ is an elementary path, and $\zeta(X)=0$ otherwise. This inhomogeneous term comes from the $Y=\emptyset$ term in the $X=\emptyset$ component equation. $\mathscr{K}$ is the remaining linear operator.

The main step in RuelLE's method [1] is to define a Banach space topology in an appropriate subspace of $\mathscr{E}$, such that $\mathscr{K}$ be a bounded operator in this subspace. Eq. (2.11) then has a unique solution in that space, defined by iteration, for $|z|\|\mathscr{K}\|<1$. We now show how this can be done in the present case. Let $\Delta(\omega)$ be a strictly positive translation invariant function of one elementary path, to be chosen later. Let $\Delta(X)$ be the product of the $\Delta(\omega)$ for all elementary paths that constitute $X$. Then, the subspace of those measurable $h \in \mathscr{E}$ for which:

$$
\operatorname{Sup}_{X} \Delta(X)^{-1}|h(X)|=\|h\|<\infty
$$

is a Banach space $\mathscr{E}_{\Delta} \subset \mathscr{E}$, with (2.12) as the definition of the norm. We now give bounds on $\mathscr{K}$. We first consider the case of real potentials $\varphi_{\perp}$ and $\varphi_{\|}$and define $\phi_{+}, \phi_{-}, M$, and $M_{0}$ by $(1.2,3,5)$. Let $h \in \mathscr{E}_{\Delta}$. From (1.5) and the definitions, we obtain:

$|\mathscr{K} h(X+\omega)| \leqq \Delta(X)\|h\| \exp \left(\beta \phi_{-}\right) \cdot \exp \left\{\int|d \bar{\omega}||K(\omega, \bar{\omega})| \Delta(\bar{\omega})\right\}$.

By the same method as in the continuous case, one shows easily [4c, d] that:

$$
\begin{gathered}
\int|d \bar{\omega}||K(\omega, \bar{\omega})| \Delta(\bar{\omega}) \leqq \sum_{j \geqq 1} \int\left|P_{00}^{j \beta}(d \bar{\omega})\right| \Delta(\bar{\omega}) \\
\cdot\left[\frac{1}{j} v(\omega, \bar{\omega})+\beta \phi_{+}+e^{\beta \phi-}-1\right]
\end{gathered}
$$

where $v(\omega, \bar{\omega})$ is the number of lattice sites that are of the form $\omega(t)$ $-\bar{\omega}(t+(k-1) \beta)$ for some $t \in(0, \beta)$ and some $k(1 \leqq k \leqq j)$. The previous description of the Poisson process makes it clear that for almost all $\omega$ and $\bar{\omega}$ :

$$
v(\omega, \bar{\omega}) \leqq j(n(\omega)+1)+n(\bar{\omega})
$$


where $n(\omega)$ is the number of jumps of the path $\omega$. This suggest to look for $\Delta(\omega)$ in the form:

$$
\Delta(\omega)=\xi \exp [\tau n(\omega)]
$$

where $\xi>0, \tau>0$. From (2.15) and (1.30), we obtain:

$$
\begin{aligned}
& \int|d \bar{\omega}||K(\omega, \bar{\omega})| \Delta(\bar{\omega}) \leqq \sum_{j \geqq 1} \xi^{j} \exp \left[j \beta\left(M e^{\tau}+M_{0}\right)\right] \\
& \cdot\left\{n(\omega)+\beta M e^{\tau}+\beta \phi_{+}+e^{\beta \phi-}\right\} .
\end{aligned}
$$

Therefore, if $\xi, \tau$ and $\varphi_{\perp}$ satisfy the inequality:

or equivalently:

$$
\tau>\sum_{j}\left(\xi \exp \left[\beta\left(M e^{\tau}+M_{0}\right)\right]\right)^{j}
$$

$$
\xi<\frac{\tau}{\tau+1} \exp \left[-\beta\left(M e^{\tau}+M_{0}\right)\right]
$$

then $\mathscr{K}$ is a bounded operator in $\mathscr{E}_{\Delta}$ and satisfies:

$$
\begin{aligned}
\|\mathscr{K}\| \leqq & \xi^{-1} \exp \left\{\beta \phi_{-}+\sum_{j} \xi^{j} \exp \left[j \beta\left(M e^{\tau}+M_{0}\right)\right]\right. \\
& \left.\cdot\left(\beta M e^{\tau}+\beta \phi_{+}+e^{\beta \phi_{-}}\right)\right\} .
\end{aligned}
$$

Elimination of $\xi$ in the exponent with the help of (2.19) gives the simpler bound:

$$
\|\mathscr{K}\| \leqq \xi^{-1} \exp \left[\beta M \tau e^{\tau}+\beta \phi_{-}+\tau\left(\beta \phi_{+}+\exp \left(\beta \phi_{-}\right)\right)\right] .
$$

Similar bounds are obtained for complex $\varphi_{\perp}, \varphi_{\|} \in \mathscr{B}$. One has to replace $\phi_{+}, \phi_{-}$and $M_{0}$ by $0, \phi$ and $\operatorname{Re} M_{0}$ respectively. More precisely:

Lemma 1. Let $\mathscr{E}_{\Delta}$ be defined by $(2.12,16)$, for fixed $\xi$ and $\tau$, with $\tau>0$ and $0<\xi<1$. Let $\mathscr{D}_{0}(\xi, \tau) \subset \mathscr{B}$ be the (open connected) subset of those $\varphi_{\perp} \in \mathscr{B}$ for which

$$
\xi<\frac{\tau}{\tau+1} \exp \left[-\beta\left(M e^{\tau}+\operatorname{Re} M_{0}\right)\right] .
$$

Then $\mathscr{K}$ is a bounded operator in $\mathscr{E}_{\Delta}$ for all $\left(\varphi_{\perp}, \varphi_{\|}\right) \in \mathscr{D}_{0}(\xi, \tau) \times \mathscr{B}$, and satisfies $\|\mathscr{K}\| \leqq R^{-1}$, where;

$$
R=\xi \exp \left\{-\left[\beta M \tau e^{\tau}+\beta \phi+\tau \exp (\beta \phi)\right]\right\} .
$$

In the same conditions, $\mathscr{K}$ is not only bounded, but even analytic [11].

Lemma 2. Let $\mathscr{E}_{\Delta}$ and $\mathscr{D}_{0}(\xi, \tau)$ be defined as in lemma 1. Then $\mathscr{K}$ is norm analytic in $\left(\varphi_{\perp}, \varphi_{\|}\right)$for $\left(\varphi_{\perp}, \varphi_{\|}\right) \in \mathscr{D}_{0}(\xi, \tau) \times \mathscr{B}$.

Sketch of the Proof. From $(2.7,11), \mathscr{K}$ can be decomposed as the product of two operators. The first one is multiplication by functions of the form $\exp [-F(\omega, X)]$. It is independent of $\varphi_{\perp}$ and norm analytic in $\varphi_{\|} \in \mathscr{B}$. The second one is the operator $L$ defined by

$$
L h(X+\omega)=f d Y K(\omega, Y) h(X+Y) .
$$


By the same methods as above, one can then prove that $L$, as a function of $\varphi_{\perp}$ and $\varphi_{\|}$, has the following properties:

(1) $L$ is Fréchet-differentiable [11] with respect to $\varphi_{\|}$.

(2) $L$ is Fréchet-differentiable with respect to $\varphi_{\perp}$. This is proved most easily by expressing the measures $P^{t}(d \omega)$ associated with two transverse potentials $\varphi_{\perp}$ and $\varphi_{\perp}^{\prime} \in \mathscr{B}$ in terms of the positive measure associated with the potential $-\operatorname{Max}\left(\left|\varphi_{\perp}(r)\right|,\left|\varphi_{\perp}^{\prime}(r)\right|\right)$, with respect to which the former are absolutely continuous.

(3) From bounds used in the proof of (1) and (2), it follows that $L$ is jointly continuous in $\varphi_{\perp}$ and $\varphi_{\|}$.

Lemmas 1 and 2 are the starting point for the proof of the results in Section 3. In the previous estimates, and therefore in all subsequent results, we have used the simple bound (1.30). Slightly better results can be obtained if one uses instead the better bound (1.29).

\section{Results}

In this section, we state without proof the results relative to the previous quantum lattice. The proofs are identical to or simpler than those of the continuous case [4c]. The new ingredients are essentially contained in Section 2.

Theorem 1. Define $\mathscr{E}_{\Delta}$ by $(2.12,16)$ for fixed $\xi$, $\tau$. Let $\mathscr{D} \equiv \mathscr{D}(\xi, \tau)$ $\subset \mathbb{C} \times \mathscr{B} \times \mathscr{B}$ be the set of those $\left(z, \varphi_{\perp}, \varphi_{\|}\right)$for which $\varphi_{\perp} \in \mathscr{D}_{0}(\xi, \tau)$ and $|z|<R$, as defined in lemma 1 . Then, for $\left(z, \varphi_{\perp}, \varphi_{\|}\right) \in \mathscr{D}$ :

(i) The Eq. (2.11) has a unique solution $\varrho_{\Lambda}$ in $\mathscr{E}_{\Delta}$, obtained by iteration. The solution is norm analytic in $\left(z, \varphi_{\perp}, \varphi_{\|}\right)$for $\left(z, \varphi_{\perp}, \varphi_{\|}\right) \in \mathscr{D}$. The solution coincides with (2.4) within $\mathscr{D}$, and satisfies;

$$
\left\|\varrho_{\Lambda}\right\| \leqq \frac{|z|}{\xi}\left(1-\frac{|z|}{R}\right)^{-1}
$$

(ii) The infinite volume equation;

$$
\varrho=\zeta+z \mathscr{K} \varrho
$$

has a unique solution in $\mathscr{E}_{\Delta}$, which is norm analytic in $\left(z, \varphi_{\perp}, \varphi_{\|}\right)$within $\mathscr{D}$ and satisfies the same inequality (3.1) as $\varrho_{\Lambda} \cdot \varrho$ is translation invariant.

(iii) Let $\Lambda$ be fixed. We say that $\Lambda^{\prime} \supset \Lambda$ becomes infinite if the distance $D$ of $\Lambda$ to the complement of $\Lambda^{\prime}$ tends to infinity. Then, when $D$ tends to infinity, $\left\|A_{\Lambda} \varrho_{\Lambda^{\prime}}-A_{\Lambda} \varrho\right\|$ tends to zero. The convergence is uniform with respect to $\Lambda$ for fixed $\left(z, \varphi_{\perp}, \varphi_{\|}\right) \in \mathscr{D}$, and uniform in $z$ for $|z| \leqq R^{\prime}<R$, for fixed $\left(\varphi_{\perp}, \varphi_{\|}\right)$.

Analyticity in $\varphi$ implies that if $\varphi=\varphi_{0}+\sum_{i=1}^{n} z_{i} \varphi_{i}$ with $\varphi_{0}, \ldots, \varphi_{n} \in \mathscr{B}$, then $\varrho_{\Lambda}$ (and $\varrho$ ) is norm analytic in $z_{1}, \ldots, z_{n}$. In particular, this implies analyticity with respect to the components $\varphi(r)$ of $\varphi$. Uniformity of the 
convergence in (iii) with respect to $\varphi_{\perp}$ and $\varphi_{\|}$could also be included. This would require that $\varphi_{\perp}$ stays away from the boundary of $\mathscr{D}_{0}(\xi, \tau)$ and that both $\varphi_{\perp}$ and $\varphi_{\|}$satisfy uniform restrictions on their decrease at infinity.

Theorem 2. For $\left(z, \varphi_{\perp}, \varphi_{\|}\right) \in \bigcup_{\xi, \tau} \mathscr{D}(\xi, \tau)=\overline{\mathscr{D}}$, the density matrices (2.3) tend to well defined limits in the sense that;

$$
\operatorname{Sup}_{r_{i}, s_{j} \in \Lambda}\left|\bar{\varrho}_{\Lambda^{\prime}}\left(r^{m}, s^{m}\right)-\bar{\varrho}\left(r^{m}, s^{m}\right)\right| \rightarrow 0
$$

when $\Lambda^{\prime}$ becomes infinite in the previous sense. Both $\bar{\varrho}_{\Lambda}$ and $\bar{\varrho}$ are analytic functions of $\left(z, \varphi_{\perp}, \varphi_{\|}\right)$in $\overline{\mathscr{D}}$ (either for fixed arguments or in the Sup. norm). For $\left(z, \varphi_{\perp}, \varphi_{\|}\right) \in \mathscr{D}(\xi, \tau)$, they satisfy the inequality;

$$
\left|\bar{\varrho}_{(\Lambda)}\left(r^{m}, s^{m}\right)\right| \leqq \frac{|z|}{\xi}\left(1-\frac{|z|}{R}\right)^{-1} m ! \tau^{m}
$$

with $R$ given by (2.23). $\varrho$ is invariant by the group of discrete translations that leave the lattice invariant.

The definition of $\bar{\varrho}$ and the bound (3.5) follow from (2.3) and its infinite volume analogue. Notice that the analyticity of $\bar{\varrho}_{\Lambda}$ and $\bar{\varrho}$ is defined in a way independent of $(\xi, \tau)$ and holds therefore in $\overline{\mathscr{D}}$, whereas the analyticity of $\varrho_{\Lambda}$ and $\varrho$ in Theorem 1 was defined within $\mathscr{E}_{\Delta}$ and therefore held in the corresponding $\mathscr{D}(\xi, \tau)$.

We now consider the pressure.

Theorem 3. The function $\beta^{-1} V^{-1} \log Z_{\Lambda}$ is an analytic function of $\left(z, \varphi_{\perp}, \varphi_{\|}\right)$within $\overline{\mathscr{D}}$. When $\Lambda$ becomes infinite, it converges to a function $p\left(z, \varphi_{\perp}, \varphi_{\|}\right)$which is analytic in $\overline{\mathscr{D}}$. For fixed $\varphi_{\perp} \in \mathscr{D}_{0}(\xi, \tau), \varphi_{\|} \in \mathscr{B}$, the convergence is uniform in $z$ for $z \leqq R^{\prime}<R$, where $R$ is given by (2.23). For real potentials and real positive $z, p\left(z, \varphi_{\perp}, \varphi_{\|}\right)$coincides with the usual grand canonical pressure. For $\left(z, \varphi_{\perp}, \varphi_{\|}\right) \in \overline{\mathscr{D}}, \quad \psi \in \mathscr{B}, p\left(z, \varphi_{\perp}, \varphi_{\|}\right)$ satisfies;

$$
\begin{aligned}
\beta z \frac{d p}{d z} & =\bar{\varrho}(0,0) \\
\left.\frac{d}{d \lambda} p\left(z, \varphi_{\perp}, \varphi_{\|}+\lambda \psi\right)\right|_{\lambda=0} & =-\frac{1}{2} \sum_{r} \psi(r) \bar{\varrho}(0, r ; 0, r), \\
\left.\frac{d}{d \lambda} p\left(z, \varphi_{\perp}+\lambda \psi, \varphi_{\|}\right)\right|_{\lambda=0} & =-\frac{1}{2} \sum_{r} \psi(r)[\bar{\varrho}(0, r)-\bar{\varrho}(0,0)] .
\end{aligned}
$$

This implies in particular the convergence of the Mayer expansion of $p$ as a power series in $z$ for $|z|<R$. One can also expand $p$ as a convergent functional power series in $\varphi_{\perp}$ and $\varphi_{\|}$(cf. [3]).

We now define cluster matrices $\bar{\chi}\left(r^{m}, s^{m}\right)$ by the relation [4b]:

$$
\bar{\varrho}(R, S)=\sum_{\left\{R_{i}\right\}} \sum_{\left\{S_{i}\right\}} \prod_{i} \bar{\chi}\left(R_{i}, S_{i}\right)
$$


where $R=r^{m}, S=s^{m}$, and the sums run over all possible partitions of $R$ and $S$ into families of subsets $\left\{R_{i}\right\}$ and $\left\{S_{i}\right\}$. The RDM satisfy a cluster property in the following sense:

Theorem 4. For $\left(z, \varphi_{\perp}, \varphi_{\|}\right) \in \overline{\mathscr{D}}$, the functions $\bar{\chi}(R, S)$ are absolutely summable functions of the differences of their arguments. More precisely, let $\varphi_{\perp} \in \mathscr{D}_{0}(\xi, \tau)$. Let $\gamma$ be positive; define $\eta, \eta(\gamma)$ and $R(\gamma)$ by;

$$
\begin{aligned}
\eta & =\xi \exp \left[\beta\left(M e^{\tau}+\operatorname{Re} M_{0}\right)\right], \\
\frac{\eta}{1-\eta} & =\frac{\eta(\gamma)}{1-\eta(\gamma)}+\gamma \frac{\eta(\gamma)}{[1-\eta(\gamma)]^{2}} \\
R(\gamma) & =R \eta(\gamma) \eta^{-1}
\end{aligned}
$$

so that $0<\eta(\gamma)<\eta<\frac{\tau}{\tau+1}, R(\gamma)<R$, and $R(\gamma)$ increases to $R$ when $\gamma$ decreases to zero. $R$ is defined as previously by (2.23). Then, for any $\gamma>0$ and any $z$ such that $|z|<R(\gamma)$, the following holds;

$$
\begin{gathered}
\sum_{R, S}|\bar{\chi}(R, S+0)| \leqq\left(\frac{|z|}{\gamma R(\gamma)}\right)^{m-1}|z| \exp \left[\beta\left(M e^{\tau}+\operatorname{Re} M_{0}\right)\right] \\
\cdot\left(1-\frac{|z|}{R(\gamma)}\right)^{-1}\left(1-\eta(\gamma) \frac{|z|}{R(\gamma)}\right)^{-1}
\end{gathered}
$$

All previous results have been stated with $R$ defined by (2.23). This definition, as well as that of $\mathscr{D}_{0}(\xi, \tau)$ through $(2.22)$ was appropriate to describe the joint analyticity properties in $\left(z, \varphi_{\perp}, \varphi_{\|}\right)$. For fixed physical, i.e. real, $\varphi_{\perp}$ and $\varphi_{\|}$, the analyticity domain in $z$ is improved if one uses (2.21) with (2.19) replaced by an equality. On then obtains:

$$
\begin{aligned}
R_{1}= & \frac{\tau}{\tau+1} \exp \left\{-\left[\beta\left(M(\tau+1) e^{\tau}+M_{0}\right)+\beta \phi_{-}+\right.\right. \\
& \left.\left.+\tau\left[\beta \phi_{+}+\exp \left(\beta \phi_{-}\right)\right]\right]\right\} .
\end{aligned}
$$

One can then take the maximum of this quantity for $\tau>0$. All the results in theorems 1 to 4 that do not involve analyticity in $\varphi_{\perp}, \varphi_{\|}$, hold for $|z|<R_{1}$.

Let now the $C^{*}$-algebra $\mathfrak{A}$ of the observables of the system be defined as in ref. [5]. From the previous results, we deduce the following.

Theorem 5. $\bar{\varrho}$ defines an extremal translation invariant state on $\mathfrak{A}$ for $\varphi_{\|}$and $\varphi_{\perp}$ real in $\mathscr{B}, z$ real positive, and $z<R_{1}$ for some $\tau$, where $R_{1}$ is given by (3.14).

The extremality of the state follows from the weak clustering property $[12,13]$ expressed by theorem 4 . The system is then in a single thermodynamic phase.

Theorem 6. All previous results (theorems 1 to 5) hold with z replaced everywhere by

$$
z^{-1} \exp \left[\beta \sum_{r}\left(\varphi_{\|}(r)-\varphi_{\perp}(r)\right)\right] .
$$

This follows from the symmetry between empty sites and occupied sites, and can be seen most easily in the spin language, where it corresponds to changing the sign of the magnetic field. 


\section{Conclusion}

We first compare the results for the quantum lattice with other cases.

1. Comparison with the Continuous Quantum Case [4]. The lattice case turns out to be substantially simpler. The discreteness of the configuration space considerably reduces the topological difficulties of the problem, while the greater simplicity of the Poisson process enables us to give explicit lower bounds on the radius of convergence of the $z$ expansions, whereas in the corresponding continuous case (Bose statistics with hard cores), similar bounds were defined implicitly by complicated integral equations.

The most interesting simplification in the lattice case comes from the boundedness of the kinetic energy operator $T$. It is straightforward to extend the functional integral representation of the statistical operator to the case where $T$ is non positive and even non hermitian, and to obtain analyticity properties with respect to the transverse, as well as the longitudinal potential, and the fugacity. These properties imply analyticity with respect to the temperature, so that the integral representation also applies to the time evolution operator $e^{i t H}$. This method will be used elsewhere to study the Green functions of the system. This situation is in sharp contrast with that in the continuous case, where the corresponding problem of going from the Wiener to the Feymann integral is much more difficult.

2. Comparison with the Classical Lattice Case. A new phenomenon occurs in the latter. Because of the discreteness of the integration space, one can improve the K.S. equations by subtracting out part of the contribution of the hard core, thereby obtaining a domain of convergence of the $z$ expansion which includes a neighborhood of the positive real $z$ axis for $\beta$ sufficiently small. Here, however, we have again a continuous integration space, in the sense that each point has measure zero, so that the analogous improvement does not occur in an obvious way. We are therefore unable to prove that the domain of analyticity covers the positive real $z$ axis for $\beta$ sufficiently small, thereby excluding the occurrence of phase transitions at sufficiently high temperature [14] $]^{1}$.

We next list some other models which can be treated by the same method.

(1) We have considered Bose statistics. One can as well consider the simpler cases of particles obeying Boltzmann or Fermi statistics, with the same kinetic energy operator, as defined in (1.10), and the same interactions. In the former case, only the term $j=1$ survives in the path

1 This further result has been obtained subsequently by G. Gallavotrit, S. Miracle-Sole and D. Robinson (Private communication). 
integration (2.6), as well as in (2.3). One obtains the same results as in the Bose case, but now the lower bound on the radius of convergence is given, for real $\varphi_{\perp}$ and $\varphi_{\|}$, by:

where:

$$
\begin{aligned}
R_{1}= & \tau \exp \left\{-\left[\beta\left(M(\tau+1) e^{\tau}+M_{0}\right)+\beta \phi_{-}\right.\right. \\
& \left.\left.+\tau\left(1+\beta \phi_{+}+\phi_{-} \frac{e^{\beta c}-1}{c}\right)\right]\right\}
\end{aligned}
$$

$$
c=-\operatorname{Min}_{r} \varphi_{\|}(r) .
$$

In the Fermi case, the hard core in $\varphi_{\|}$plays no role, the presence of more than one particle on a given site being already excluded by the Pauli principle. Therefore in (2.14) the term $v(\omega, \bar{\omega})$ drops out and one can take $\Delta(\omega)=\xi$. One then gets the following expression for the lower bound on the radius of convergence of the $z$ expansion:

$R_{1}=\frac{\tau}{\tau+1} \exp \left\{-\left[\beta\left(M+M_{0}\right)+\beta \phi_{-}+\tau\left(\beta \phi_{+}+e^{\beta \phi-}-1\right)\right]\right\}$

where $\xi$ and $\tau$ are now related by:

$$
\xi=\frac{\tau}{\tau+1} \exp \left[-\beta\left(M+M_{0}\right)\right] .
$$

(2) We have considered interactions with point hard cores. One can also take a potential without hard core: $\varphi_{\|}(0)<\infty$. A sufficient condition for stability is then:

$$
\varphi_{\|}(0) \geqq-\sum_{r \neq 0} \operatorname{Min}\left(\varphi_{\|}(r), 0\right)=\phi_{-} .
$$

The same results hold, with again the additional simplification that one can take $\Delta(\omega)=\xi$. The analyticity properties in $\varphi_{\|}$hold only with respect to the components $\varphi_{\|}(r)$ for $r \neq 0$, for fixed $\varphi_{\|}(0)$ and $\sum_{r \neq 0}\left|\varphi_{\|}(r)\right| \leqq \varphi_{\|}(0)$. The lower bound on the radius of convergence of the $z$ expansion for real $\varphi_{\perp}$ and $\varphi_{\|}$becomes in the Bose case:

$$
\begin{aligned}
R_{1}= & \frac{\tau}{\tau+1} \exp \left\{-\left[\beta\left(M+M_{0}\right)+\beta \phi_{-}+\tau \beta \phi_{+}\right.\right. \\
& \left.\left.-\frac{\phi-}{c} \log \left[1-\tau\left(e^{\beta c}-1\right)\right]\right]\right\}
\end{aligned}
$$

where $c$ is defined by (4.2); $\tau$ and $\xi$ are related by (4.4), with the additional restriction that $1+\tau\left(1-e^{\beta c}\right)>0$. Furthermore, $\phi_{+}$now contains $\varphi_{\|}(0)$. In the Fermi case, one can use either (4.3) or (4.6), with $\varphi_{\|}(0)$ absent from $\phi_{+}$.

(3) Conversely, one can consider interactions with extended hard cores. This brings back some of the difficulties associated with hard cores in the continuous case. The same results hold, but the bounds on the radius of convergence become more complicated. 
In the models listed under (2) and (3) one loses the symmetry between empty and occupied sites, and therefore the extension of the results to the high $z$ region.

Acknowledgements. I am grateful to Dr. D. RoBInson for a stimulating discussion which revived my interest in this problem and to Dr. S. VaradHaN for enlightening explanations on stochastic processes. I wish to thank Prof. K. SymanzIK and Prof. W. Zimmermans for the hospitality extended to me at the Courant Institute of Mathematical Sciences, where this work was done.

\section{References}

1. Ruelle, D.: Ann. Phys. 25, 109 (1963).

2. HILL, T. L.: Statistical mechanics. New York: McGraw Hill 1956.

3. Gallavotti, G., and S. Mrracle-Sole: Commun. Math. Phys. 7, 274 (1968).

4. Grnibre, J.: (a) J. Math. Phys. 6, 238 (1965); (b) 252 (1965); (c) 1432 (1965);

(d) Proceedings of the IUPAP Conference on Statistical Mechanics. New York: Benjamin 1967.

5. Robinson, D.: Commun. Math. Phys. 6, 151 (1967).

6. - Commun. Math. Phys. 7, 337 (1968).

7. Streater, R. F.: Commun. Math. Phys. 6, 233 (1967).

8. Matsubara, T., and H. Matsuda: Prog. Theor. Phys. (Kyoto) 16, 569 (1956).

9. KAC, M.: Probability and related topics in physical sciences. New York: Interscience 1959.

10. Dоов, J. L.: Stochastic processes, Chap. VIII. New York: Wiley 1953.

11. HILle, E., and R. S. PhIllips: Functional analysis and semi groups. A. M. S. Providence (1957). Section (3-17).

12. Kastler, D., and D. Robinson: Commun. Math. Phys. 3, 151 (1966).

13. Ruelle, D.: Commun. Math. Phys. 3, 133 (1966).

14. Gallavotti, G., S. Miracle-Sole, and D. Robinson: Phys. Letters 25A, 433 (1967).

J. GINIBRE

Laboratoire de Physique Théorique

Faculté des Sciences

F 91 Orsay 